\title{
Investigation of the forced spatial vibrations of a wood shaper, caused by rotor's unbalance of its electric motor and by cutting forces
}

\author{
Valentin Slavov ${ }^{1, *}$ and Georgi Vukov ${ }^{2}$ \\ ${ }^{1}$ University of Chemical Technology and Metallurgy, Department of Applied Mechanics, 1756 Sofia, Bulgaria \\ ${ }^{2}$ University of Forestry, Faculty of Forest Industry, 1756 Sofia, Bulgaria
}

\begin{abstract}
Mechanic-mathematical matrix modeling of the forced spatial vibrations of a wood shaper is performed in this study. The wood shaper is modeled as a mechanical system of three rigid bodies, which are connected by elastic and damping elements with each other and with the motionless floor. This mechanical system has 18 degrees of freedom. Formulas and algorithms are developed for computer calculating, analysis and synthesis of designing and investigating of this machine. This study renders an account the geometric, kinematic, mass, inertia, elastic and damping properties of the machine. A system of differential equations is derived. Analytical solutions are presented. The study presnts results of the numerical investigations of the forced spatial vibrations by using parameters of a particular machine. They allow to select parameters that reduce harmful vibrations for people and constructions.
\end{abstract}

\section{Introduction}

Some vibrations occur during the operation of the wood shapers. They affect service staff, the construction of the machine and the quality of the treated wood surfaces [1]. These vibrations are generated by several sources, but the most important are the rotor's unbalance of drive electric motor and the cutting forces. The presence of unbalance of the electric motor's rotor causes polyharmonic vibrations in the radial direction.

Wear and damage of the cutting tools of woodworking shapers change the characteristics of the forces of their interaction with the processing material [2]. This affects performance of the machines and impairs the accuracy and quality of their production [9]. Uneven wear or damage of the tool and accumulation of superposition in separate parts of the instrument have main influence. They strongly affect the cutting force and create additional variable loads during the operation of the wood shapers $[4,5]$. These loads are transmitted to the spindle and by its two bearing units reach the other elements and the machine's body. Specific studies for investigating the effect of the uneven wear and the damage of the cutting tool on the cutting forces and the machine's work are required [3]. The machine can be seen as a mechanical vibrating system with known characteristics in this studies [6,7].

The purpose of this work is to perform a mechanic mathematical modeling in the 3D space of the forced spatial vibrations of a wood shaper. Formulas and algorithms for analysis and synthesis, which are applicable in designing and investigating of wood shapers, should be developed. Geometric, kinematic, mass, inertial, elastic, damping properties and force actions of this mechanical system should be taken into account [8].

\section{Dynamic model}

The dynamic model is shown in Figure 1.

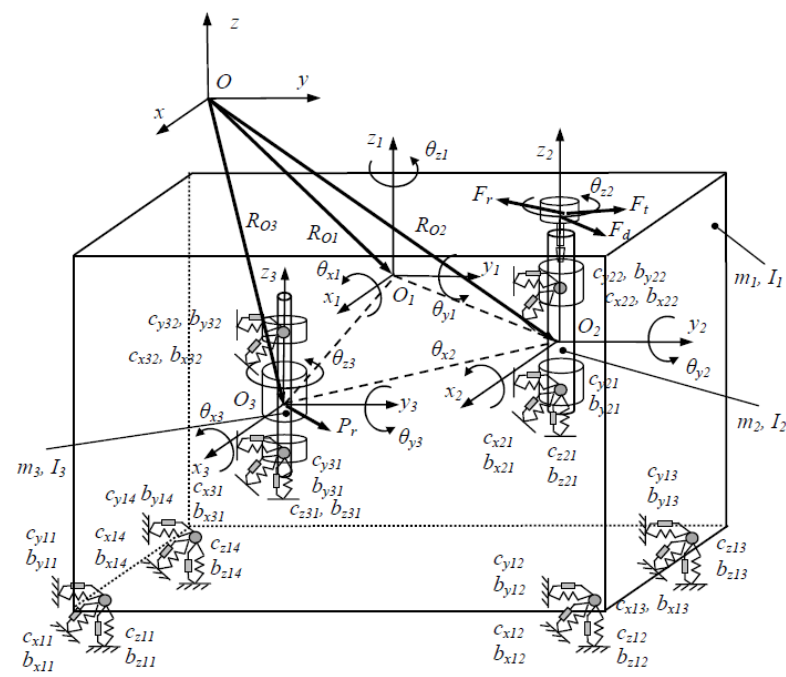

Fig. 1. Dynamic model.

It is assumed that the mechanical system consists of three rigid bodies: 1 - shaper's body; 2 - spindle; 3 rotor of the driving electric motor. The shaper's body is mounted to a foundation by means of 4 elastic-damping elements. Their linearized coefficients of elasticity and

\footnotetext{
Corresponding author: valslavov@abv.bg
} 
damping are $\quad c_{x 1 k}, c_{y 1 k}, c_{z 1 k} ; \kappa=1 \div 4, \quad$ and $b_{x 1 k}, b_{y 1 k}, b_{z 1 k} ; \kappa=1 \div 4$. The spindle is mounted to the shaper's body by means of 2 elastic-damping elements (bearings). Their linearized coefficients of elasticity and damping are $c_{x 2 k}, c_{y 2 k}, c_{z 2 k} ; \kappa=1,2 \quad$ and $b_{x 2 k}, b_{y 2 k}, b_{z 2 k} ; \kappa=1,2$. The rotor of the driving electric motor is mounted to the body via 2 bearings, which are elastic-damping elements, too. Their linearized coefficients of elasticity and damping are $c_{x 3 k}, c_{y 3 k}, c_{z 3 k} ; \kappa=1,2$ and $b_{x 3 k}, b_{y 3 k}, b_{z 3 k} ; \kappa=1,2$. The figure shows the local coordinate systems and the reference coordinate system which coincides with the coordinate system of body 1 and in which all the vectors are projected.

It is assumed that the axes of the local coordinate systems are parallel to the axes of the reference coordinate system.

The bodies are characterized by their masses $m_{i}$ and their tensors of mass inertia moments $\mathbf{I}_{\boldsymbol{\Theta} \Theta}^{\mathbf{i}}$.

$$
\mathbf{I}_{\Theta \Theta}^{\mathbf{i}}=\left[\begin{array}{ccc}
I_{O x x} & -I_{O x y} & -I_{O x z} \\
-I_{O y x} & I_{O y y} & -I_{O y z} \\
-I_{O z x} & -I_{O z y} & I_{O z z}
\end{array}\right] ; i=1,2,3
$$

The three bodies of the mechanical system perform spatial vibrations - three small translations and three small rotations relative to the axes of the rectangular local coordinate systems that are fixed to the bodies.

The position of the mechanical system in the space is defined by the generalized coordinate vector which is

$$
\begin{gathered}
\mathbf{q}=\left[\begin{array}{lllllllll}
x_{1} & y_{1} & z_{1} & \theta_{x 1} & \theta_{y 1} & \theta_{z 1} & x_{2} & y_{2} & z_{2} \\
\theta_{x 2} & \theta_{y 2} & \theta_{z 2} & x_{3} & y_{3} & z_{3} & \theta_{x 3} & \theta_{y 3} & \theta_{z 3}
\end{array}\right]^{\mathrm{T}}
\end{gathered}
$$

The mechanical system has 18 degrees of freedom.

\section{Forced spatial vibrations}

The differential equations which describe the forced vibrations are deduced by using the Lagrange's method.

$$
\frac{\mathrm{d}}{\mathrm{dt}}\left(\frac{\partial E_{K}}{\partial \dot{\mathbf{q}}}\right)-\left(\frac{\partial E_{K}}{\partial \mathbf{q}}\right)+\frac{\partial E_{D}}{\partial \dot{\mathbf{q}}}+\frac{\partial E_{P}}{\partial \mathbf{q}}=\mathbf{Q}
$$

where $E_{K}$ and $E_{P}$ are respectively the kinetic and the potential energy of the mechanical system, $E_{D}$ is the energy of dissipation or dissipative function and $\mathbf{Q}$ is the vector of generalized forces.

The obtained system of differential equations which describes the forced vibrations of the investigated mechanical system is

$$
\mathbf{M}_{18 \times 18} \cdot \ddot{\mathbf{q}}_{18 \times 1}+\mathbf{B}_{18 \times 18} \cdot \dot{\mathbf{q}}_{18 \times 1}+\mathbf{C}_{18 \times 18} \cdot \mathbf{q}_{18 \times 1}=\mathbf{Q}_{18 \times 1}
$$

The matrix in these equations which characterizes the mass-inertial properties of the mechanical system is $\mathbf{M}$.
$\mathbf{B}$ is the matrix that characterizes the damping properties of this system and $\mathbf{C}-$ its elastic properties.

\subsection{Kinetic energy}

The kinetic energy of the mechanical system is calculated by the formula

$$
E_{K}=\sum_{i=1}^{3} E_{K i}
$$

where

$$
\begin{aligned}
& E_{K i}=\frac{1}{2} \cdot\left(\mathbf{m}_{\mathbf{R R}}^{\mathbf{i}} \cdot \mathbf{V}_{\mathbf{C i}}^{\mathbf{0}}{ }^{\mathrm{T}} \cdot \mathbf{V}_{\mathbf{C i}}^{\mathbf{0}}+\mathbf{\Omega}_{\mathbf{i}}^{\mathbf{i} \mathrm{T}} \cdot \mathbf{I}_{\mathbf{\Theta} \Theta}^{\mathbf{i}} \cdot \mathbf{\Omega}_{\mathbf{i}}^{\mathbf{i}}\right) ; \\
& \mathbf{m}_{\mathbf{R R}}^{\mathbf{i}}=\int_{V_{i}} \rho_{i} \cdot \mathbf{I} \cdot d V_{i}=m_{i} \cdot \mathbf{I} ; \quad \mathbf{I}=\left[\begin{array}{cccc}
1 & 0 & 0 & 0 \\
0 & 1 & 0 & 0 \\
0 & 0 & 1 & 0 \\
0 & 0 & 0 & 1
\end{array}\right]
\end{aligned}
$$

$\mathbf{V}_{\mathbf{C i}}^{\mathbf{0}}$ - vector of the linear velocity of the point $C i$ (the mass center of body $i$ ), projected in the reference coordinate system;

$\mathbf{\Omega}_{\mathbf{i}}^{\mathbf{i}}$ - vector of the angular velocity of the body $i$, projected in the local coordinate system.

The elements of the matrix $\mathbf{M}$ are defined by the expression

$$
m_{i, j}=\frac{\partial^{2} E_{K}}{\partial \dot{q}_{i} \cdot \partial \dot{q}_{j}}
$$

\subsection{Potential energy}

The potential energy of the mechanical system is calculated by the formula

$$
\begin{aligned}
E_{P}= & \left(\sum_{k=1}^{4} \frac{1}{2} c_{k} \cdot\left(\mathbf{\delta}_{\mathbf{k}}^{01}\right)^{2}+\sum_{k=1}^{2} \frac{1}{2} c_{k} \cdot\left(\boldsymbol{\delta} \mathbf{r}_{\mathbf{k}}^{12}\right)^{2}+\sum_{k=1}^{2} \frac{1}{2} c_{k} \cdot\left(\mathbf{\delta r}_{\mathbf{k}}^{13}\right)^{2}\right)+ \\
& +\sum_{i=1}^{3}-m_{i} \cdot \mathbf{g}^{\mathrm{T}} \cdot \mathbf{R}_{\mathbf{C i}}^{\mathbf{0}}
\end{aligned}
$$

where

$c_{k}$ - elasticity coefficient;

$\mathbf{R}_{\mathbf{C i}}^{\mathbf{0}}$ - vector of the position of the mass center in the reference coordinate system;

$\boldsymbol{\delta} \mathbf{r}_{\mathbf{k}}^{\mathbf{0 1}}$ - the deformation of the elastic elements between the base (denoted conditionally by "0") and body 1 ;

$\delta \mathbf{r}_{\mathbf{k}}^{12}$ - deformation of the elastic elements between body 1 and body 2 ;

$\delta \mathbf{r}_{\mathbf{k}}^{\mathbf{1 3}}$ - deformation of the elastic elements between body 1 and body 3 ;

$\mathbf{g}=\left[\begin{array}{llll}0 & 0 & g & 0\end{array}\right]^{\mathrm{T}}$ is a vector of the gravitational acceleration;

$k$ is the number of the elastic elements between two bodies of the mechanical system. 
The elements of the matrix $\mathbf{C}$ are defined by the expression

$$
c_{i, j}=\frac{\partial^{2} E_{P}}{\partial q_{i} \cdot \partial q_{j}}
$$

\subsection{Energy of dissipation (dissipative function)}

Dissipative function is calculated by the formula

$$
F_{b}=\sum \frac{1}{2} \cdot b_{k} \cdot\left(\boldsymbol{\delta} \dot{\mathbf{r}}_{\mathbf{k}}\right)^{2}
$$

where $\delta \dot{\mathbf{r}}_{\mathbf{k}}$ is the velocity of deformation of the elastic elements.

The elements of the matrix $\mathbf{B}$ are obtained by replacing the elements of the matrix $\mathbf{C}-c_{i, j}$ with $b_{i, j}$.

$$
\mathbf{B}=\left[b_{i, j}\right]
$$

\subsection{Generalized forces}

The vector of generalized forces has the form

$$
\begin{aligned}
\mathbf{Q}= & {\left[\begin{array}{lllllll}
0 & 0 & 0 & 0 & 0 & 0 & \mathbf{Q}_{\mathbf{F} 2(3 \times 1)}{ }^{\mathrm{T}} \\
& \mathbf{Q}_{\mathbf{Q} 2(3 \times 1)}{ }^{\mathrm{T}} & \mathbf{Q}_{\mathbf{F} 3(3 \times 1)}{ }^{\mathrm{T}} & \mathbf{Q}_{\mathbf{Q} 3(3 \times 1)}{ }^{\mathrm{T}}
\end{array}\right]^{\mathrm{T}} }
\end{aligned}
$$

where

$$
\mathbf{Q}_{\mathbf{F} 2}=\mathbf{Q}_{\mathbf{F d}}+\mathbf{Q}_{\mathbf{F c}} ; \quad \mathbf{Q}_{\mathbf{Q} 2}=\mathbf{Q}_{\mathbf{Q d}}+\mathbf{Q}_{\mathbf{Q c}}
$$

The unbalance force of the cutting tool from its uneven wear is

$$
\begin{aligned}
& \mathbf{Q}_{\mathbf{F d}}=\left[\begin{array}{c}
F_{x} \\
F_{y} \\
0
\end{array}\right] ; F_{x}=F_{d} \cdot \cos (\omega \cdot t) ; F_{y}=F_{d} \cdot \sin (\omega \cdot t) \\
& \mathbf{Q}_{\mathbf{Q d}}\left(F_{d}\right)=\mathbf{U}_{\mathbf{1}}^{\mathbf{\Omega 0 T}} \cdot\left(\tilde{\mathbf{r}}_{\mathbf{P} 2}^{\mathbf{0}} \cdot \mathbf{Q}_{\mathbf{F d}}\right)
\end{aligned}
$$

where

$$
\begin{aligned}
\mathbf{U}_{1}^{\mathbf{\Omega 0} \mathrm{T}} & =\left[\begin{array}{ccc}
1 & 0 & \theta_{y 1} \\
0 & 1 & -\theta_{x 1} \\
0 & \theta_{x 1} & 1
\end{array}\right]^{\mathrm{T}} ; \\
\tilde{\mathbf{r}}_{\mathbf{P 2}}^{\mathbf{T}} & =\left[\begin{array}{ccc}
0 & l_{P 2 z}^{0} & -l_{P 2 y}^{0} \\
-l_{P 2 z}^{0} & 0 & l_{P 2 x}^{0} \\
l_{P 2 y}^{0} & -l_{P 2 x}^{0} & 0
\end{array}\right] ; \\
\mathbf{r}_{\mathbf{P 2}}^{\mathbf{0}} & =\left[\begin{array}{l}
l_{P x 2}^{0} \\
l_{P y 2}^{0} \\
l_{P z 2}^{0}
\end{array}\right]=\left[\begin{array}{l}
l_{x 1}^{0}+l_{x 2}^{1}+l_{P x 2} \\
l_{y 1}^{0}+l_{y 2}^{1}+l_{P y 2} \\
l_{z 1}^{0}+l_{z 2}^{1}+l_{P z 2}
\end{array}\right] ;
\end{aligned}
$$

$$
\mathbf{r}_{\mathbf{P} 2}=\left[\begin{array}{l}
l_{P x 2} \\
l_{P y 2} \\
l_{P z 2}
\end{array}\right] .
$$

Cutting force is a distributed force acting on the cutting edge of the tool. This force is replaced by its resultant force, whose application point $S$ is in the middle of the cutting edge. Resultant force's two components - tangential and radial - are used in practice. Each of these forces also creates a moment relative to the reference coordinate system. Thus the generalized force includes the generated forces and moments from the cutting forces.

The generalized forces of the components of the cutting force are

$$
\mathbf{Q}_{\mathbf{F c}}=\left[\begin{array}{c}
\frac{F_{t}}{2}+\frac{F_{t}}{2} \cdot \cos (6 . \omega . t)+\frac{F_{r}}{2}+\frac{F_{r}}{2} \cdot \cos (6 . \omega . t) \\
\frac{F_{t}}{2}+\frac{F_{t}}{2} \cdot \sin (6 . \omega \cdot t)+\frac{F_{r}}{2}+\frac{F_{r}}{2} \cdot \sin (6 . \omega . t) \\
0
\end{array}\right]
$$

where

$F_{t}$ - tangential component of the cutting force;

$F_{r}$ - radial component of the cutting force.

The generalized moments of the components of the cutting force are

$\mathbf{Q}_{\mathbf{Q c}}\left(F_{c}\right)=\mathbf{U}_{\mathbf{1}}^{\mathbf{\Omega 0 T}} \cdot\left(\tilde{\mathbf{r}}_{\mathbf{S} \mathbf{2}}^{\mathbf{T}} \cdot \mathbf{Q}_{\mathbf{F c}}\right)$,

where

$\mathbf{M}\left(F_{c}\right)=\tilde{\mathbf{r}}_{\mathbf{S} \mathbf{2}}^{\mathbf{T}} \cdot \mathbf{Q}_{\mathbf{F c}}-$ moment of cutting forces;

$$
\begin{aligned}
& \mathbf{U}_{\mathbf{1}}^{\mathbf{\Omega 0} \mathrm{T}}=\left[\begin{array}{ccc}
1 & 0 & \theta_{y 1} \\
0 & 1 & -\theta_{x 1} \\
0 & \theta_{x 1} & 1
\end{array}\right]^{\mathrm{T}} ; \\
& \tilde{\mathbf{r}} \mathbf{S 2}^{\mathbf{T}}=\left[\begin{array}{ccc}
0 & l_{S 2 z}^{0} & -l_{S 2 y}^{0} \\
-l_{S 2 z}^{0} & 0 & l_{S 2 x}^{0} \\
l_{S 2 y}^{0} & -l_{S 2 x}^{0} & 0
\end{array}\right] ; \\
& \mathbf{r}_{\mathbf{S 2}}^{\mathbf{0}}=\left[\begin{array}{l}
l_{S x 2}^{0} \\
l_{S y 2}^{0} \\
l_{S z 2}^{0}
\end{array}\right]=\left[\begin{array}{l}
l_{x 1}^{0}+l_{x 2}^{1}+l_{S x 2} \\
l_{y 1}^{0}+l_{y 2}^{1}+l_{S y 2} \\
l_{z 1}^{0}+l_{z 2}^{1}+l_{S z 2}
\end{array}\right] ; \\
& \mathbf{r}_{\mathbf{S 2}}=\left[\begin{array}{l}
l_{S x 2} \\
l_{S y 2} \\
l_{S z 2}
\end{array}\right]-.
\end{aligned}
$$

The unbalance force of the motor's rotor is

$\mathbf{Q}_{\mathbf{F} 3}=\left[\begin{array}{c}P_{r x 3} \\ P_{r y 3} \\ 0\end{array}\right] ; \mathbf{Q}_{\mathbf{Q} 3}\left(P_{r}\right)=\mathbf{U}_{\mathbf{i}}^{\mathbf{\Omega 0 T}} \cdot\left(\tilde{\mathbf{r}}_{\mathbf{P i}}^{\mathbf{T}} \cdot \mathbf{Q}_{\mathbf{F} 3}\right)$ 
where

$$
\begin{aligned}
& P_{r x 3}=P_{1} \cdot \cos (\omega t)+P_{2} \cdot \cos (3 \omega t)+P_{3} \cdot \cos (5 \omega t) \\
& P_{r y 3}=P_{1} \cdot \sin (\omega t)+P_{2} \cdot \sin (3 \omega t)+P_{3} \cdot \sin (5 \omega t) \\
& \mathbf{U}_{\mathbf{i}}^{\mathbf{2 0 T}}=\left[\begin{array}{ccc}
1 & 0 & \theta_{y 1} \\
0 & 1 & -\theta_{x 1} \\
0 & \theta_{x 1} & 1
\end{array}\right]^{\mathrm{T}} \\
& \tilde{\mathbf{r}}_{\mathbf{P} 3}^{\mathbf{T}}=\left[\begin{array}{ccc}
0 & l_{P z 3}^{0} & -l_{P y 3}^{0} \\
-l_{P z 3}^{0} & 0 & l_{P x 3}^{0} \\
l_{P y 3}^{0} & -l_{P x 3}^{0} & 0
\end{array}\right]
\end{aligned}
$$

\subsection{Solutions of the differential equations}

The general solutions of the system of differential equations in matrix form, with initial conditions $t=0, \quad q(0)=q_{0}, \quad \dot{q}(0)=\dot{q}_{0}$ and harmonic disturbing forces are

$$
\begin{aligned}
& q(t)=\sum_{r=1}^{18} \frac{2}{g_{r}^{2}+h_{r}^{2}}\left[\mathbf{G}_{r} \cdot \mathbf{M} \cdot \dot{q}(0)+\left(-\alpha_{r} \cdot \mathbf{G}_{r} \cdot \mathbf{M}+\beta_{r} \cdot \mathbf{H}_{r} \cdot \mathbf{M}+\mathbf{G}_{r} \cdot \mathbf{B}\right) \cdot q(0)\right] \cdot e^{-\alpha_{r} t} \cdot \cos \beta_{r} t+ \\
& +\sum_{r=1}^{18} \frac{2}{g_{r}^{2}+h_{r}^{2}}\left[\mathbf{H}_{r} \cdot \mathbf{M} \cdot \dot{q}(0)+\left(-\alpha_{r} \cdot \mathbf{H}_{r} \cdot \mathbf{M}-\beta_{r} \cdot \mathbf{G}_{r} \cdot \mathbf{M}+\mathbf{H}_{r} \cdot \mathbf{B}\right) \cdot q(0)\right] \cdot e^{-\alpha_{r} t} \cdot \sin \beta_{r} t+ \\
& +\operatorname{Re}\left\{\sum_{k=0}^{n} \sum_{r=1}^{18} \frac{2}{g_{r}^{2}+h_{r}^{2}} \cdot \frac{\alpha_{r} \cdot \mathbf{G}_{r}+\beta_{r} \cdot \mathbf{H}_{r}+i \cdot k \cdot \Omega \cdot \mathbf{G}_{r}}{\omega_{r}^{2}-k^{2} \cdot \Omega^{2}+i \cdot 2 \cdot k \cdot \sigma_{r} \cdot \omega_{r} \cdot \Omega} \mathbf{Q} \cdot e^{i k \Omega t_{3}}\right\}
\end{aligned}
$$

where

$$
\begin{aligned}
g_{r}= & -2 \cdot \alpha_{r}\left(\mathbf{V}_{r}^{\mathrm{T}} \cdot \mathbf{M} \cdot \mathbf{V}_{r}-\mathbf{W}_{r}^{\mathrm{T}} \cdot \mathbf{M} \cdot \mathbf{W}_{r}\right)- \\
& -4 \cdot \beta_{r} \cdot \mathbf{V}_{r}^{\mathrm{T}} \cdot \mathbf{M} \cdot \mathbf{W}_{r}+\mathbf{V}_{r}^{\mathrm{T}} \cdot \mathbf{B} \cdot \mathbf{V}_{r}-\mathbf{W}_{r}^{\mathrm{T}} \cdot \mathbf{B} \cdot \mathbf{W}_{r} ; \\
h_{r}= & 2 \cdot \beta_{r}\left(\mathbf{V}_{r}^{\mathrm{T}} \cdot \mathbf{M} \cdot \mathbf{V}_{r}-\mathbf{W}_{r}^{\mathrm{T}} \cdot \mathbf{M} \cdot \mathbf{W}_{r}\right)- \\
& -4 \cdot \alpha_{r} \cdot \mathbf{V}_{r}^{\mathrm{T}} \cdot \mathbf{M} \cdot \mathbf{W}_{r}+2 \cdot \mathbf{V}_{r}^{\mathrm{T}} \cdot \mathbf{B} \cdot \mathbf{W}_{r} ; \\
\mathbf{G}_{r}= & g_{r} \cdot \mathbf{L}_{r}+h_{r} \cdot \mathbf{R}_{r} ; \\
\mathbf{L}_{r}= & \mathbf{V}_{r} \cdot \mathbf{V}_{r}^{\mathrm{T}}-\mathbf{W}_{r} \cdot \mathbf{W}_{r}^{\mathrm{T}} ; \\
\mathbf{H}_{r}= & h_{r} \cdot \mathbf{L}_{r}-g_{r} \cdot \mathbf{R}_{r} ; \\
\mathbf{R}_{r}= & \mathbf{V}_{r} \cdot \mathbf{W}_{r}^{\mathrm{T}}+\mathbf{W}_{r} \cdot \mathbf{V}_{r}^{\mathrm{T}} .
\end{aligned}
$$

\section{Results and discussion}

A dynamic synthesis of a particular wood shaper has been performed. Mass, inertial and geometric parameters of details and units of this wood shaper are determined by means of a CAD program [10].

Table 1 gives the mass-inertial, elastic and damping characteristics, the geometric parameters of the mechanical system as well as the disturbing force and the coordinates of the point where it is applied.

The forced vibrations of this mechanical system are calculated by developed program in a mathematical software environment [11]. Analytical and graphical results are obtained with this program.

Several numerical calculations are performed and illustrated. The purpose of these studies is to select such characteristics of the elastic and damping elements of the wood shaper fastening that can minimize the damping time of the vibrations and amplitudes of forced vibrations to be less than those allowed in the standards in wood industry. 
Table 1. Parameters of the mechanical system.

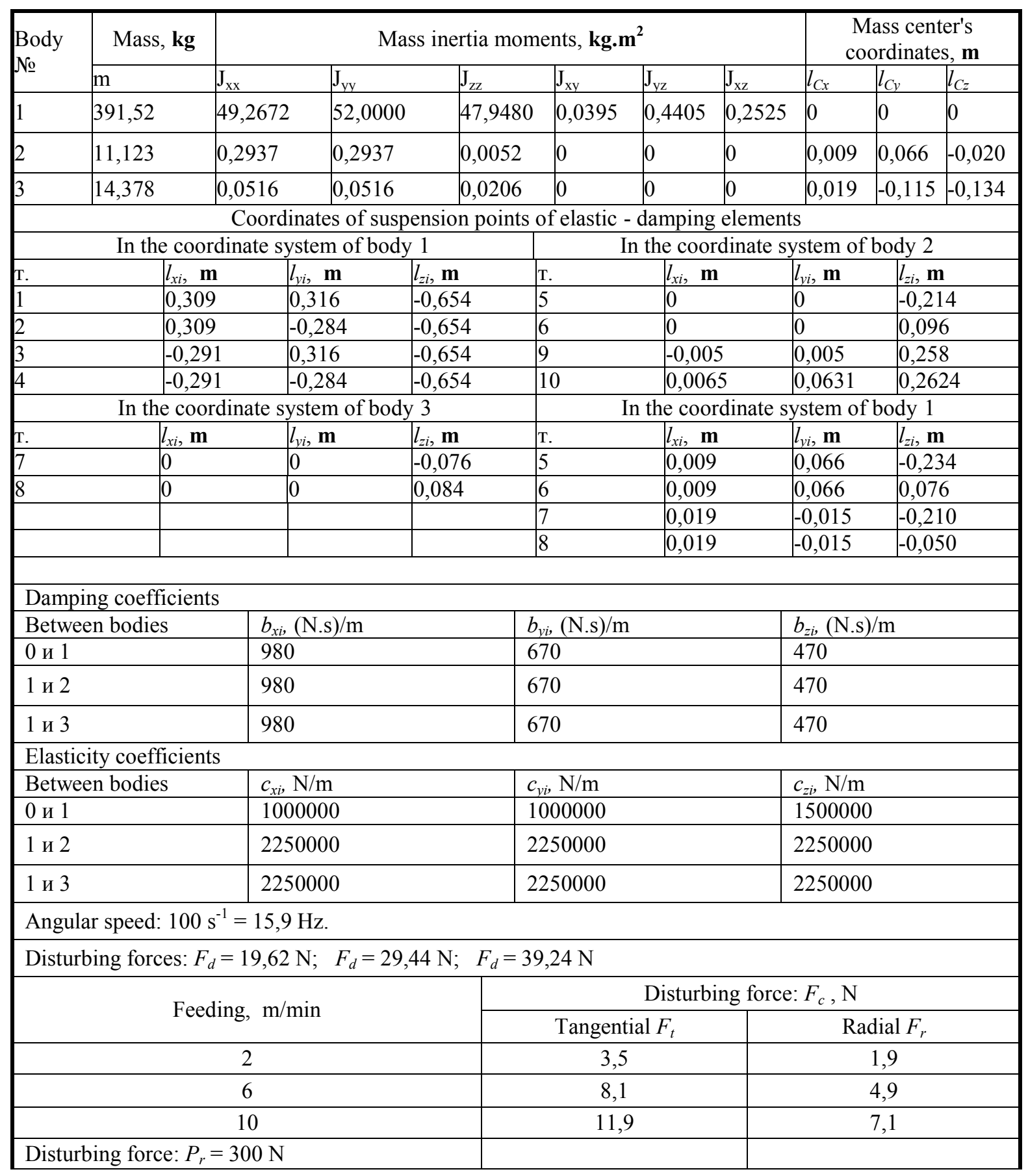

The elastic-damping elements are marked with points 1 to 8 . The application point of the disturbing force $F_{d}$, which coincides with the mass center of the tool, is marked by point $9(P)$. The application point of the cutting force $F_{c}$ is marked by point $10(S)$. It is in the middle of the cutting edge of the tool.

The vibrations on all 18 generalized coordinates of this mechanical system are obtained as a result of the provided investigations. Due to the limited volume of the article, only a few of them are illustrated here. The linear vibrations on the three coordinates of the machine's body, the rotor of the electric motor and the spindle are presented. The results are given at angular speed $100 \mathrm{~s}^{-1}$, feed $10 \mathrm{~m} / \mathrm{min}$ and disturbing force 39,24 N. 9 variants are investigated. The amplitudes of the considered coordinates are 

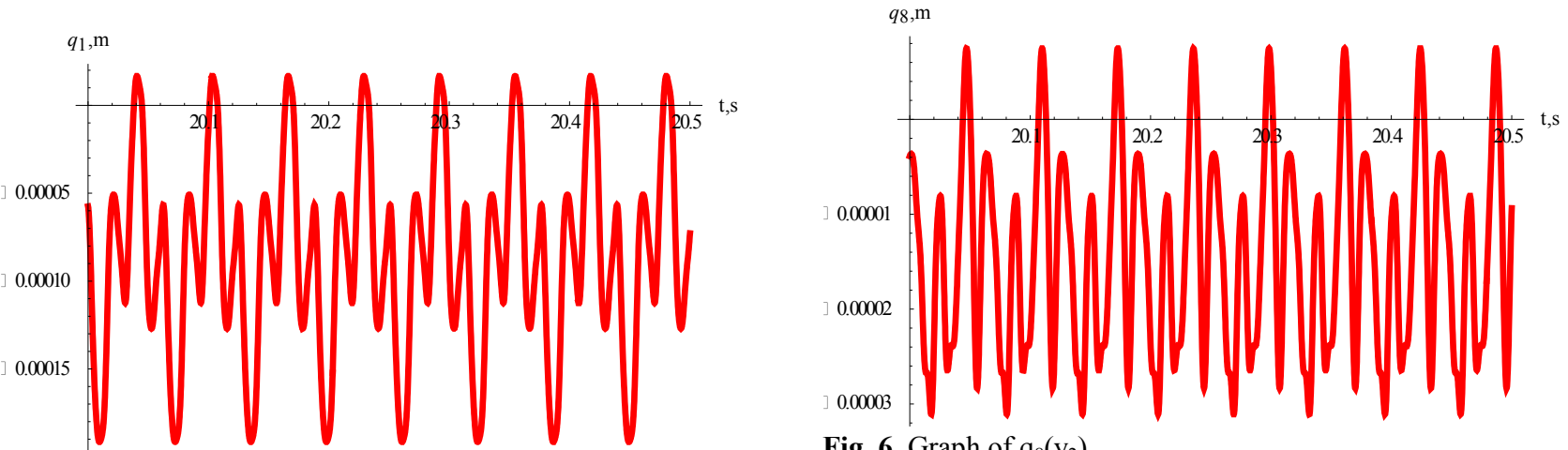

Fig. 2. Graph of $\mathrm{q}_{1}\left(\mathrm{x}_{1}\right)$.

Fig. 6. Graph of $\mathrm{q}_{8}\left(\mathrm{y}_{2}\right)$.
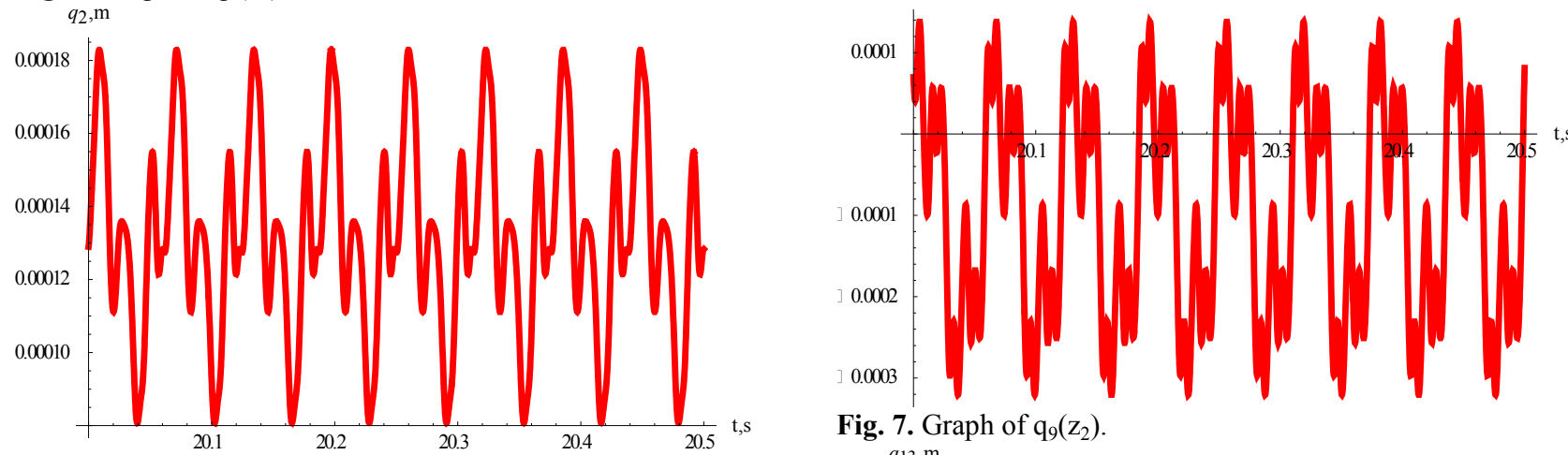

Fig. 3. Graph of $\mathrm{q}_{2}\left(\mathrm{y}_{1}\right)$.

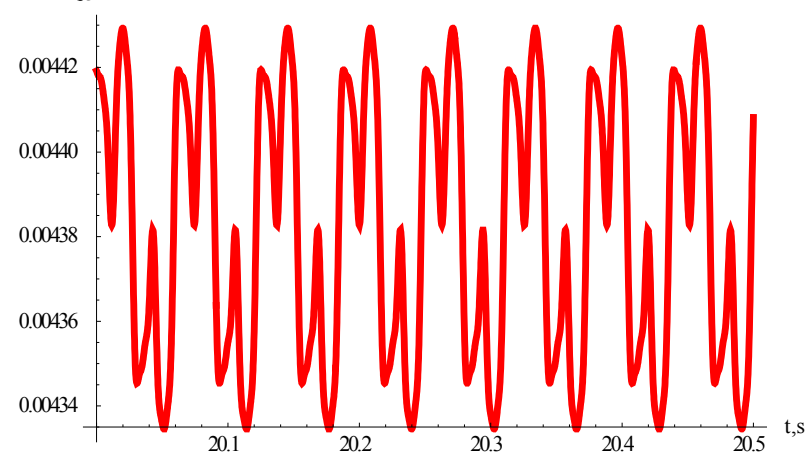

Fig. 7. Graph of $\mathrm{q}_{9}\left(\mathrm{z}_{2}\right)$.

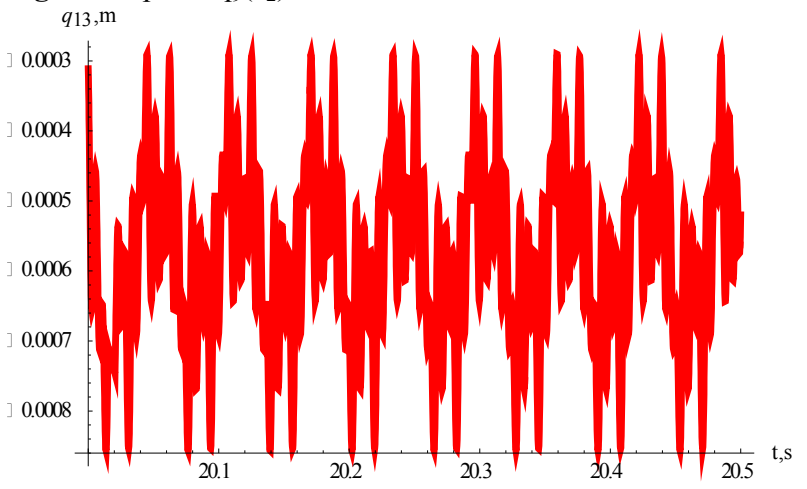

Fig. 4. Graph of $\mathrm{q}_{3}\left(\mathrm{z}_{1}\right)$
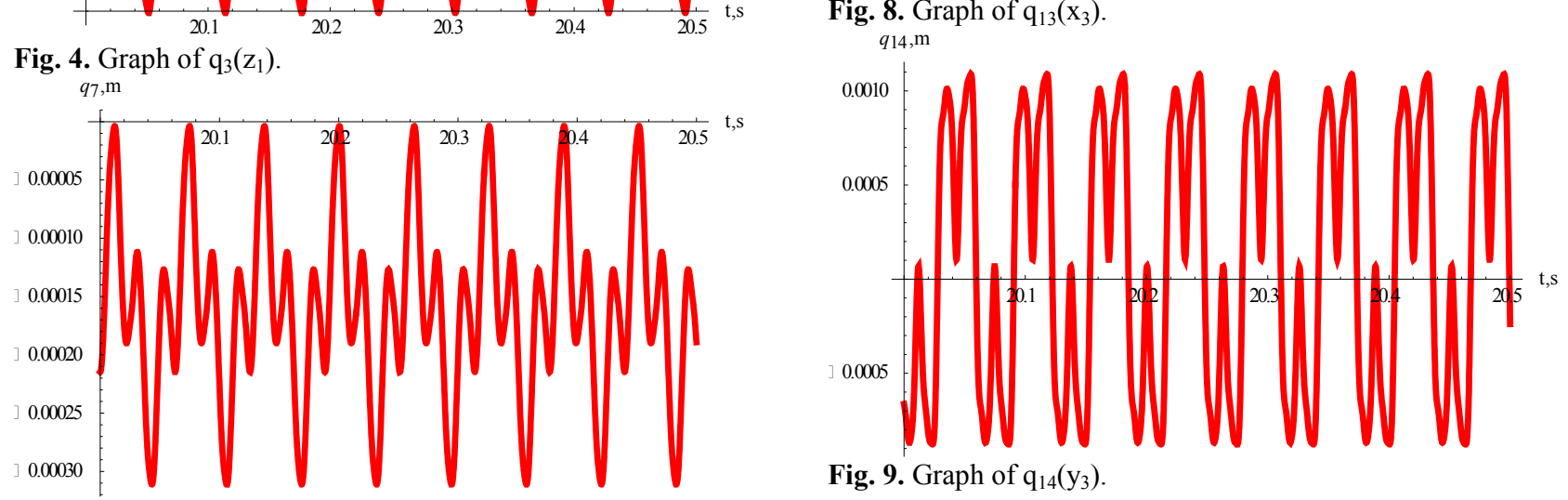

Fig. 5. Graph of $\mathrm{q}_{7}\left(\mathrm{x}_{2}\right)$.

Fig. 9. Graph of $\mathrm{q}_{14}\left(\mathrm{y}_{3}\right)$. 


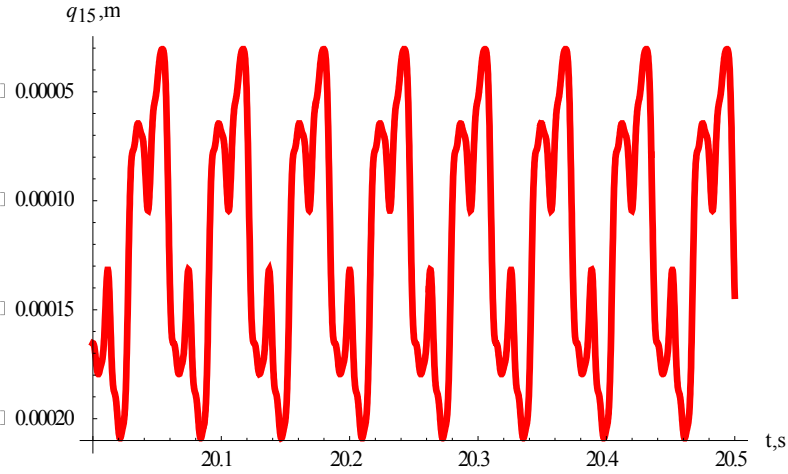

Fig. 10. Graph of $\mathrm{q}_{15}\left(\mathrm{z}_{3}\right)$.

The analysis of the results shows that the presence of unbalance of the electric motor's rotor causes spatial vibrations not only on the motor but also on the entire machine. The specific values of these vibrations depend on the machine's parameters and its operating conditions. The amplitudes of the forced vibrations increase as the unbalanced force rises. This affirms the need for monitoring the status of the electric motor to keep the limits of vibrations allowed by the standard.

\section{Conclusion}

The paper presents a study of the forced spatial vibrations of a woodworking shaper caused by the unbalance of the electric motor's rotor. The differential equations of its forced vibrations are deduced. The obtained results in the study of kinematics, dynamics, free undamped vibrations and free damped vibrations of this machine are used. The mass-inertial, elastic and damping characteristics, the geometric parameters of the mechanical system as well as the disturbing force are taken into account. Analytical solutions have been received. Some numerical calculations are performed with the parameters of a certain wood shaper. The obtained results are presented graphically and they are analyzed. This analysis confirms the need of accurate monitoring the status of the electric motor to keep the limits of machine's vibrations allowed by the standard.

\section{References}

1. ISO 2631-1:1997. Mechanical vibration and shockEvaluation of human exposure to whole-body vibration-Part 1: General requirements

2. C. Prakasvudhisarn, S. Kunnapapdeelert, P. Yenradee (2009): Optimal cutting condition determination for desired surface roughness in end milling. The International Journal of Advanced Manufacturing Technology, 41: pp. 440-451.

3. G. Keturakis, I. Juodeikiene, (2007): Investigation of Milled Wood Surface Roughness. Materials science 13(1): pp. 47-51.

4. G. Vukov, V. Atanasov, V. Slavov, Zh. Gochev (2018): Investigation of Spatial Vibrations of a Wood Milling Shaper and its Spindle, Caused by Cutting Force, Proceedings of the $5^{\text {th }}$ International Conference on Processing Technologies for the Forest and Bio-based Products Industries (PTF BPI 2018) Freising/Munich: pp. $144 \div 152$.

5. G. Vukov, V. Slavov, P. Vichev, Zh. Gochev (2019), Investigations of the Free Space Vibrations of a Woodworking Shaper, Considered as a Mechanical System with Three Main Bodies, Proceedings $4^{\text {th }}$ International Scientific Conference "Wood Technology \& Product Design", Ohrid, Republic of Macedonia, pp. 127- 135.

6. I. Angelov, V. Slavov, Matrix Mechanics. Dynamics and Vibrations, Problems, (Sofia, Avangard Prima, 2010) (in Bulgarian)

7. I. Angelov, Matrix Theory of Vibration in Engineering, (Sofia, Avangard Prima, 2012) (in Bulgarian)

8. I. Angelov, V. Slavov, Matrix Mechanics. Symbol Programming, (Sofia, Avangard Prima, 2014) (in Bulgarian)

9. M. Rousek, Z. Kopecký, M. Svatoš (2010): Problems of the quality of wood machining by milling stressing the effect of parameters of machining on the kind of wood. Annals of Warsaw University of Life Sciences - SGGW 72 No 72: pp. 233-242.

10. Software product: Solid Works

11. Wolfram S. Mathematica: A System for Doing Mathematics by Computer. Reading, Addison Wesley, (1988) 